\title{
HIGH RESOLUTION ADAPTIVE ARRAYS BASED ON RANDOM PROCESSING TECHNIQUES: FREQUENCY HOPPPING MODULATION
}

\author{
Montse Nájar, Miguel A. Lagunas. \\ Department of Signal Theory and Communications \\ Universitat Politècnica de Catalunya \\ 08071 BARCELONA, SPAIN \\ Phone:34-3-4017051. Fax: 34-3-4016447.
}

\begin{abstract}
A new architecture for adaptive arrays using Frequency Hopping modulation is addressed in this paper. The resolution of the array and the interference rejection increase substantially applying random processing to the carrier frequency of the signals. The proposed framework is composed of two different stages. The anticipative stage, devoted to minimize the noise and fixed interferences contribution and the GSLC stage which provides cancellation of follower jammers and solves the multiuser collision problem. The developed system requires neither temporal nor spatial reference for its implementation, only the frequency sequence must be known. An adaptive approach has been implemented, allowing a fast convergence to the optimal behavior.
\end{abstract}

\section{INTRODUCTION}

The resolution of an array can be increased in two different ways: swelling the number of sensors or augmenting the distances between them. Both of these options have problems. The first one raises the cost of the array and the second one has a well-known limit: if the interelement distance exceeds half of any impinging signal wave-length, grating lobes will appear in the array factor. A possible solution to this problem can be found in the nonperiodic arrays (arrays with nonequidistant elements), in this case the elements can be distributed in a deterministic or a random way. The problem of grating lobes does not appear in this kind of arrays, so the mean distance between sensors can exceed the limit of half wave-length. It is for this reason that the nonperiodic arrays allow greater resolution without incrementing the number of sensors. Some previous works dedicated to this subject are referred [1]. An important drawback of these arrays is that the level of the sidelobes can augment significantly if the number of sensors is low.

Recently, Random Sampling techniques have been developed [2]. These techniques allow to use a sampling frequency exceeding the Nyquist limit without aliasing whenever the sum of the probability density functions of all the sampling points is a constant. In this case the estimated spectrum of the randomly sampled signal will be equal to the original spectrum of the continuous signal. The output signal of an array is obtained as a combination of all the output sensors located at different positions, that is to say that an array system spatially samples the signals. Therefore, it seems to be

This work was supported by the National Research Plan of Spain, CICYT, Grant number TIC.92-0800-C05-05 and by the Copernicus Project CORELAR C8254. possible to apply some of the theoretical results obtained in the time sampling domain to the space domain in order to develop random processing methods, which permit to eliminate the grating lobes (spatial aliasing) of an array system. The output array signal consists of a vector of sensor samples (snapshot), which is taken at different instants of time. The first case to consider in Random Array Processing lies in a randomization of the sensor positions, changing them from one snapshot to another. If the probability of the sensor positioning at each point of the aperture is the same, then the mean array factor will correspond to the radiation pattern of a continuos aperture, which does not have grating lobes. Thus, the spatial aliasing has disappeared as in the temporal case, applying random techniques. The interest of this case, where the sensor position has been randomized, is more theoretical than practical, because it is unrealistic to assume that there may be mechanical shifting of the array sensors between repeating snapshots. Nonetheless, the same effect can be achieved otherwise, for instance, constructing an array with a high number of closely and equidistantly spaced elements and activating only a few of them for each snapshot. The behavior of this system, in mean, will be as if the whole array was activated, however, the cost will be lower.

Some techniques have been further studied in order to obtain a more feasible implementation, in other words, the goal is to randomize the array avoiding a mechanical displacement of the sensors. It seems that the only possibility is to randomize the frequency of the transmitted signals and this effect can be achieved using Frequency Hopping (FH) modulation. In this case, virtual sensors will appear in the aperture at random positions. Similarly to the preceding case, in order to obtain a continuous aperture, the probability of the virtual sensor positioning at each point of the aperture must be the same. FH is a method of spectrum spreading widely used to make a communication system less vulnerable in front of interferences [3]. It consist of a system in which the carrier frequency is pseudorandomly hopped over a wide band, Wss, under the control of a pseudonoise sequence. The signal bandwidth on each hop is much smaller than Wss, however, averaged over many hops, the FH signal spectrum occupies the entire spread spectrum bandwidth. Current technology permits $\mathrm{FH}$ bandwidths of the order of several $\mathrm{GHz}$ and rates greater than $1 \mathrm{Mhop} / \mathrm{sec}$. The application of $\mathrm{FH}$ modulation in an antenna array will improve substantially the SINR (Signal to Interference plus Noise Ratio) as a consequence of the increase of the resolution and the interference rejection. Nevertheless, little information is available on performance of arrays with FH signals: Compton [4] studied the adverse effects of FH modulation in an adaptive array based on the LMS (Least Mean Squared) algorithm. Bakhru [4] proposed a specific method for 
adaptive arrays using FH signals, the Maximin algorithm, which is based on the spectral characteristics of these signals, requiring neither a reference signal nor steering vectors for its implementation. Nonetheless any adaptive algorithm presents some discontinuities when used with FH modulated signals. The reason is that the changes in the signal frequency due to FH are seen by the algorithm as changes in the direction of arrival. Torrieri [6] suggested three different techniques of frequency compensation for the Maximin algorithm to solve this problem: Parameter-dependent processing is the most complicated to implement and the one which presents larger convergence. Spectral processing is the simplest to implement, but the achieved improvement is not significant. And, finally, the Anticipative processing provides the fastest convergence but exhibits the worst behavior.

This paper deals with a new architecture for FH in Array Processing, composed of two different stages. First of all the theoretical system is described, next, an adaptive approach is proposed: finally, some simulation results and conclusions will be shown.

\section{TWO STAGE RECEIVER FOR FREQUENCY HOPPING MODULATION}

It is well known that the maximum SINR criterion in Array Processing yields the optimal complex weight vector (1), both in temporal and in spatial reference systems.

$\mathbf{w}_{\text {opt }}=\mu \mathbf{R}_{\mathrm{n}}^{-1} \mathbf{s}_{\mathrm{d}}$

being $\mathbf{R}_{\mathrm{n}}$ the interference plus noise correlation matrix and $\mathbf{s}_{\mathrm{d}}$ the steering vector of the desired signal. An approach to this optimum solution is constituted of two different stages.

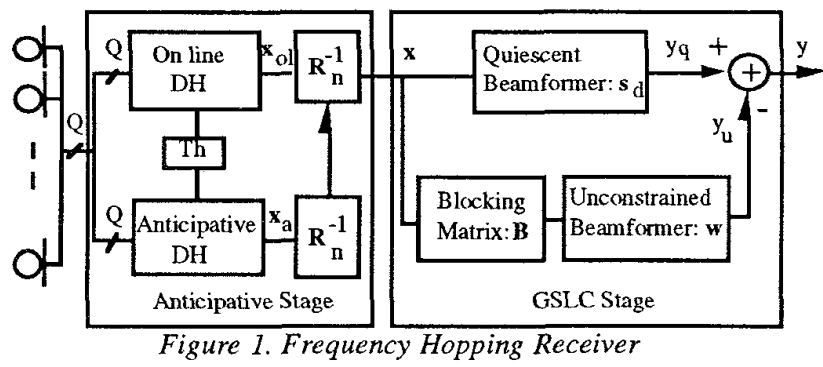

The first stage, named the Anticipative stage, is devoted to cancel interferences at fixed frequencies that are already present or active at the frequency of interest at the hop time. The second stage is considered for combating interferences that are not present at the frequency of interest at the time of the frequency hop, but may get activate sometime after the hop occurs. This is the case of adaptive jammers known as repeatback or frequency-follower jammers in military scenarios. Moreover, this problem might appear in multiuser systems, for instance, in mobile communication systems using $\mathrm{FH}$ modulation. Although users in the same cell normally use different hopping sequences, they may interfere among them when the carrier frequencies coincide in some hops. This second stage consist of a GSLC.

\subsection{Anticipative Stage}

The Anticipative stage is formed by two dehopping processors: the anticipative, which gives the name to this stage, and the on-line dehopping processors. (Figure 1).

The dehopping system (Figure 2) follows the low noise amplifier of each sensor because of bandwidth reasons in the down-conversion sequence, allowing the suppression of the noise and interferences outside the signal band.

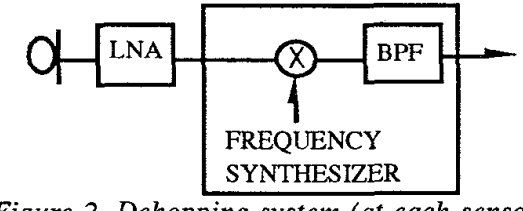

Figure 2. Dehopping system (at each sensor)

The idea of the anticipative dehopping is to obtain a previous image of the scenario in order to predict a system that maximizes the SINR at the output of the on-line dehopping as fast as possible. Thus, this dehopping is done with a carrier frequency before its transmission, $f_{h}(i+1)$. The resultant snapshot $x_{a}(n)$ contains the noise and interferences that will appear in the on-line processor in the next hopping, the desired signal at the hop frequency $f_{h}(i)$ is rejected by the dehopping with $f_{h}(i+1)$. Hence, the required $\mathbf{R}_{n}$ matrix is calculated beforehand from the anticipative dehopping output. After the hop time, the inverse of this matrix is transferred to the on-line processor multiplying the snapshot $\mathbf{x}_{\mathrm{ol}}(\mathrm{n})$, obtained by the on-line dehopping (done with the carrier frequency transmitted at each moment). This matrix blocks the scenario: noise and fixed frequency interferences, in a similar way as the blocking matrix in the GSLC stage blocks the desired signal, as it will be shown in the next section.

\subsection{Generalized Sidelobe Canceller Stage}

A weight vector equal to the steering of the desired signal $\left(\mathbf{s}_{\mathbf{d}}\right)$ must be implemented to achieve the optimal beamvector (1). The noise and fixed interferences contribution in $y_{q}(n)$ (Figure 1) are minimized. Nevertheless, new interferences that may turn up during the hop time are not canceled at this point. The second stage should maximize the SINR minimizing any directional component appearing in the array snapshot vector $\mathbf{x}(\mathbf{n})$, from angles of arrival different from the desired look direction. In conclusion, we are in front of a problem of constrained minimization power. The solution to this problem can be implemented by the so-called GSLC (Generalized Sidelobe Canceller [7]), consisting of two different paths. On the one hand, the upper path, termed the quiescent beamformer, provides the optimal solution when the input $\mathbf{x}(\mathbf{n})$ contains only white noise and the desired signal. On the other hand, the lower path attends to maximize the SINR in $y(n)$ when interferences appear in the scenario. As it is wellknown this lower path contains a blocking matrix $\mathbf{B}$ avoiding the presence of the desired signal before the unconstrained beamformer $\mathbf{w}$, which is obtained imposing a criterion of minimum mean squared error at the final output $y(n)$. 


\section{ADAPTIVE APPROACH}

The anticipative stage does not need an adaptive implementation. The inverse correlation matrix $\mathbf{R}_{\mathrm{n}}{ }^{-1}$ is calculated during the whole hop time in the anticipative processor, before to be transferred to the on-line processor, where it is kept until next hop succeed.

If the desired direction of arrival is known, the quiescent beamvector $s_{d}$ will be also known. Consequently, the only part of the receiver that would have to be adaptive is the unconstrained beamformer (Figure 1). The weight vector $w$ can be easily got by any of the adaptive algorithms that minimize the mean squared error: LMS, NLMS, RLS, ...

In a great number of applications the direction of arrival of the desired signal is unknown. In this case, the quiescent beamformer should be obtained from the snapshot $\mathbf{x}(n)$. Whenever the Anticipative stage has canceled all the interferences present in the scenario, the snapshot $\mathbf{x}(\mathrm{n})$ will contain only information about the desired signal. Thus, a simple adaptive estimation of the eigenvector corresponding to the maximum eigenvalue of the signal correlation matrix provides the quiescent weight vector $\mathbf{s}_{\mathrm{d}}$.

$\mathbf{R}_{\mathrm{i}}(\mathrm{n}+1)=\boldsymbol{\beta} \mathbf{R}_{\mathrm{i}}(\mathrm{n})+(\beta-1) \mathbf{x}(\mathrm{n}+1) \mathbf{x}^{\mathrm{H}}(\mathrm{n}+1)$

$\mathbf{v}(\mathrm{n}+1)=\mathbf{s}_{\mathrm{d}}(\mathrm{n})+\mu \mathbf{R}_{\mathrm{i}}(\mathrm{n}+1) \mathbf{s}_{\mathrm{d}}(\mathrm{n})$

$s_{d}(n+1)=\frac{v(n+1)}{v_{1}(n+1)}$

being (4) a normalization by the first component of the estimated eigenvector to have the steering vector. The correlation matrix subindex $\mathrm{i}$ indicates the hop number.

The desired signal is always present after the on-line dehopping, whatever frequency is transmitted. For this reason, it is convenient to estimate the correlation matrix from the snapshots acquired during the whole processing time, not only during the hop time. Since the snapshot $\mathbf{x}(\mathrm{n})$ depends on the hop frequency (steering vector), the correlation matrix estimation must be done in a coherent way. The correlation matrix at the Anticipative stage output, in the i-th hop, can be expressed as:

$\mathbf{R}_{\mathrm{i}}=\alpha_{\mathrm{d}}^{2} \mathbf{s}_{\mathrm{d}}\left(f_{\mathrm{h}}(\mathrm{i})\right) \mathbf{s}_{\mathrm{d}}{ }^{\mathrm{H}}\left(\mathrm{f}_{\mathrm{h}}(\mathrm{i})\right)+\mathbf{R}_{\mathrm{n}}$

When a new hop occurs the estimated correlation matrix has to be modified by a transformation matrix in order to be coherent with the incoming snapshots:

$\mathbf{R}_{\mathbf{i}+1}=\mathbf{T}_{\mathbf{j}} \mathbf{R}_{\mathbf{i}} \mathbf{T}_{\mathbf{j}}^{\mathrm{H}}$

Thereby, the correlation matrix can be adapted continuously, improving the eigenvector estimation and increasing the convergence.

Obviously the quiescent weight vector $s_{d}\left(f_{1 l}(i)\right)$ estimated at the end of each hop must also be modificd to the new one. Because it coincides with a steering vector, the frequency dependence is on the phases of its components and it is linear, as it is represented in (8). $\mathrm{Q}$ is the number of array elements.

$s_{d}\left(f_{h}(i)\right)=\left[\begin{array}{lll}1 & \exp \left(\alpha_{1} f_{h}(i)\right) & \ldots . \\ \exp \left(\alpha_{Q-1} f_{h}(i)\right)\end{array}\right]$

So, the modification consist in a simple phase multiplication by the frequency ratio: $f_{h}(i+1) / f_{h}(i)$.

From now on, it is easy to obtain the expression for the transformation matrix $\mathrm{T}_{\mathrm{i}}[8]$ :

$T_{i}=\operatorname{diag}\left[\frac{s_{d 1}\left(f_{h}(i+1)\right)}{s_{d 1}\left(f_{h}(i)\right)} \ldots \ldots \cdot \frac{s_{d Q}\left(f_{h}(i+1)\right)}{s_{d Q}\left(f_{h}(i)\right)}\right]$

Since the convergence of this estimation procedure is considerably fast, after few iterations the quiescent beamvector and the blocking matrix may be frozen. Thus, the unconstrained beamvector allows the cancellation of incoming interferences during the hop time.

\section{SIMULATION RESULTS}

The presented simulations have been made with a linear equally spaced array of 8 sensors, in which the interelement separation was half wavelength. The desired source, located at 20 degrees from the broadside direction, was a BPSK signal (4 samples/symbol) centered at $900 \mathrm{MHz}$, with $0 \mathrm{~dB}$ of SNR. This signal has been spread uniformly over a relative bandwith equal to the 50 percent, which is the ratio of the total hopping bandwith to the center frequency. The dwell time or duration of the hop interval was set equal to the duration of 9 symbols ( 36 samples). Therefore, Slow FH modulation is considered. A random sequence of 450 symbols has been generated. So, 50 frequency hops occurred.

In the first simulation only a multitone jamming is present in the scenario at 60 degrees. This interference is distributed over the spread-spectrum bandwidth, consisting of tones over half the frequency channels. The interference to noise ratio in each channel was $20 \mathrm{~dB}$. In Figure 3 the mean array factor after the 50 hops is shown with solid line. One of the array factor, particularly the correspondent to the 10-th hop is represented with dashdot line. Because in this case there is only fixed interferences, the cancellation is achieved at the output of the quiescent beamformer, being the unconstrained beamvector approximately equal to zero. In Figure 4 the evolution of the SINR is plotted over the whole time, also the SNR of each hop is calculated separately and represented in the same figure. It can be observed that they fluctuates by approximately $3 \mathrm{~dB}$.

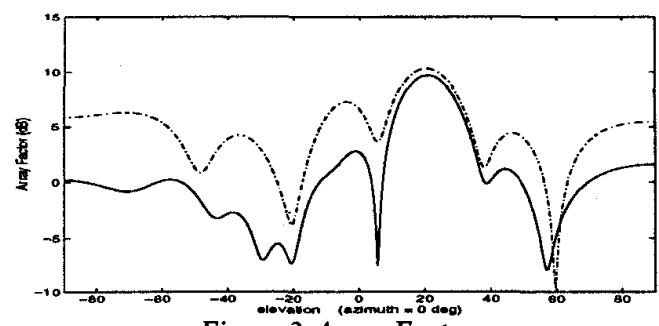

Figure 3. Array Factor 


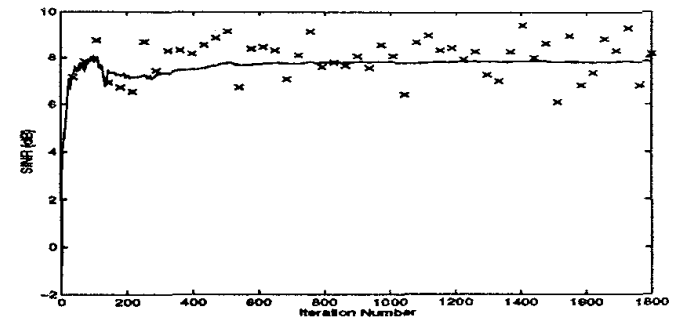

Figure 4. SINR Evolution

In the second simulation a follower jammer, radiating at -30 degrees, is added to the scenario. This interference hops with the same sequence as the desired signal with a delay of 12 samples. This signal must be canceled by the GSLC stage. The quiescent beamvector is adapted only during the three first symbols at each frequency, fixing it before the follower jammer appears at the hop frequency. Since the convergence of the algorithm is very fast, a few number of iterations are sufficient to assure the quiescent adaptation to the steering vector, and after that, the minimization of the jammer contribution by the unconstrained beamformer. The mean (solid line) and an instantaneous array factor (dashdot line) are shown in Figure 5. The evolution of the coefficients is depicted in Figure 6: a) The quiescent Beamvector (in dashdot line is plotted the theoretical steering vector), b) The unconstrained beamvector.

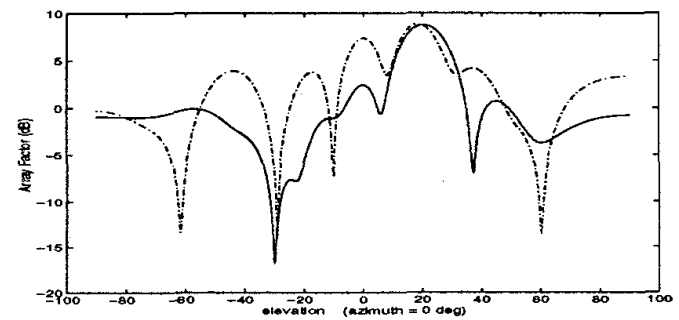

Figure 5. Array Factor
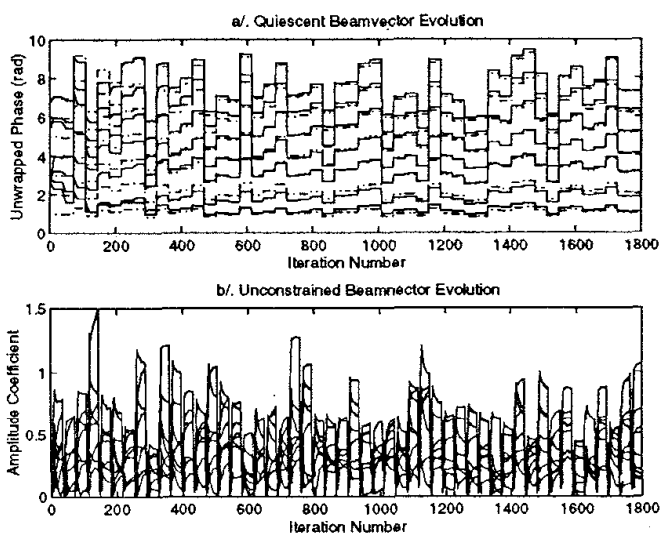

Figure 6. Coefficients Evolution

Finally, a last simulation has been done with the same array but spacing the sensors the wavelength in order to increase the resolution. If no $\mathrm{FH}$ modulation was applied the array factor would present a grating lobe at -40 degrees (Figure 7: dashdot line),being impossible to cancel an interference arriving from this direction. Using the system developed in this paper, grating lobes are reduced considerably. In Figure 7 with solid line is represented the mean array factor when an interference with a SNR of $30 \mathrm{~dB}$, at -40 degrees, is impinging the array.

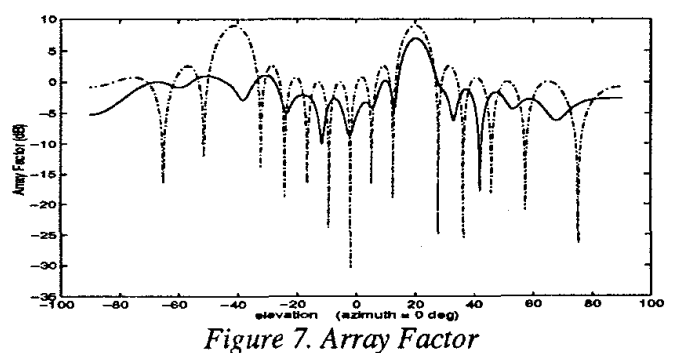

\section{CONCLUSIONS}

A new adaptive receiver for FH signals in Array Processing, has been reported in order to increase the array resolution and to improve the interference rejection. The proposed framework is composed of two different stages: The anticipative stage, devoted to cancel fixed jamers, and the GSLC stage, which minimizes the effect of the rest of interferences. An appropriate transformation of the correlation matrix ensures rapid convergence of the algorithm.

\section{REFERENCES}

[1]Steinberg B. , "Principles of Aperture and Array System Design including Random Adaptive Arrays", John Wiley \& Sons, 1976.

[2]Bilinskis I. , Lagunas M. A. , " Randomising of Array Element Spacing and of Processing Array Signals", EUSIPCO-92, vol. 3, pp. 1573-1576, Belgium, August 2427, 1992.

[3]Simon M. , Omura J. , Scholtz R. , Levit B. , "Spread Spectrum Communications", Computer Science Press, 1985.

[4]Car L. ,Compton R. ,"The Performance of an LMS Adaptive Array with Frequency Hopped Signals", IEEE Transactions on Aerospace and Electronic Systems, vol. AES-21, no. 3, pp. 360-371, May 1985.

[5]Bakhru K. , Torrieri D. , “ The Maximin Algorithm for Adaptive Arrays and Frequency-Hopping Communications", IEEE Transactions on Antennas and Propagation, vol. AP32, no. 9, pp. 919-928, September 1984.

[6]Torrieri D. , Bakhru K. ,"Frequency Compensation in an Adaptive Antenna System for Frequency-Hopping Communications", IEEE Transactions on Aerospace and Electronic Systems, vol. AES-23, no. 4, pp. 448-467, July 1987.

[7]Griffiths L1. , Buckley K. "Quiescent Pattern Control in Linearly Constrained Adaptive Arrays", IEEE Transactions on Acoustics, Speech and Signal Processing, vol. ASSP-35. no. 7, pp 917-926, July 1987.

[8]Wang H. , Kaveh M. " Coherent Signal-Subspace Processing for the Detection and Estimation of Angles of Arrival of Multiple Wide-Band Sources", IEEE Transactions on Acoustics, Speech and Signal Processing, vol. ASSP-33. no. 4, pp 823-831, August 1985. 